\title{
Electronic Stability Control of Vehicles
}

\author{
Suryakant Gupta ${ }^{1, *}$, Nidhi Hiremath ${ }^{2, * *}$, Samiksha Raut $^{2, * * *}$, Gaurav Datkhile ${ }^{3, * * * *}$, and Prasiddh Trivedi ${ }^{3, \dagger}$ \\ ${ }^{1}$ Ramrao Adik Institute of Technology, Navi Mumbai, Nerul.
}

\begin{abstract}
The paper presents the Electronic Control Unit of vehicles with the main focus on Anti-lock braking system. ABS is used in traction control and to maintain vehicle stability. The paper describes the detail study of CarSim model and it gives us the most accurate and efficient method to simulate the performance of vehicle and how ABS can be used in different scenarios. The result of vehicular model without and with ABS for different test cases is presented in the paper. The vehicular model is implemented on MATLAB Simulink environment and the corresponding results are presented.
\end{abstract}

\section{Introduction}

Electronic control unit recently has become an essential part of the vehicles as it helps prevent accidents and gives more control to the vehicle. ABS is a part of Electronic stability control. Here it plays a vital role where brakes are used in the case of emergencies by maintaining the stability of the vehicle. Hence designing a proper ABS controller is particularly important as safety of the driver is of the cardinal aspect. Testing the vehicle manually with real-time responses is not economically feasible and hence virtual environment comes into picture. The results can be thus observed by simulation[11].

CarSim by Mechanical Simulations provides the users, the environment to get responses for any type of scenarios for the vehicle. The Software can be used to customize vehicles by entering their parameters and create a path for vehicles to run. The Animator output of Carsim helps to study the vehicle movement, forces and its measurements. They can also be precisely plotted on the graph and observed[10].

\section{Description of vehicle model}

Carsim has datasets for some standard vehicles, hence from that we have a used B-class hatchback for our study but there is an option to make a vehicle from scratch in CarSim provided the parameters and dynamics of the vehicle are known. For our study we have used the same car with and without an ABS and observed the different responses[9][10].

\footnotetext{
*e-mail: suryakantgupta66@gmail.com

**e-mail: nidhihiremath@gmail.com

***e-mail: samuraut8@gmail.com

****e-mail: gaurav.datkhile@rait.ac.in

†e-mail: prasiddh.trivedi@rait.ac.in
}

\subsection{B-Class Hatchback Without ABS}

Generic B-Class (Compact) hatchback with Strut front and 5-Link rear suspension. B-Class vehicles include the Citroen C3, Ford Fiesta, Opal Corsa, and Volkswagen Polo. Compared to some of the other B-Class vehicle examples, the following has the CarSim without ABS selected on the Brakes data screen[9][10].

\subsection{B-Class Hatchback With ABS}

Generic B-Class (Compact) hatchback with Strut front and 5-Link rear suspension. B-Class vehicles include the Citroen C3, Ford Fiesta, Opal Corsa, and Volkswagen Polo. Compared to some of the other B-Class vehicle examples, the following has the CarSim ABS selected on the Brakes data screen[9][10].

\section{Literature Survey}

The friction coefficient can be expressed as an empirical function, where the wheel slip is a function argument:

$$
\mu=A .\left(B .\left(1-e^{-C s}\right)-D . s\right)
$$

Table 1. Empirical coefficient for different road type

\begin{tabular}{|l|l|l|l|l|}
\hline \multicolumn{5}{|c|}{ Road Type } \\
\hline $\begin{array}{l}\text { Empirical } \\
\text { coefficient }\end{array}$ & $\begin{array}{l}\text { Dry Con- } \\
\text { crete }\end{array}$ & $\begin{array}{l}\text { Wet Con- } \\
\text { crete }\end{array}$ & Snow & Ice \\
\hline $\mathrm{A}$ & 0.9 & 0.7 & 0.3 & 0.1 \\
$\mathrm{~B}$ & 1.07 & 1.07 & 1.07 & 1.07 \\
$\mathrm{C}$ & 0.2773 & 0.5 & 0.1773 & 0.38 \\
$\mathrm{D}$ & 0.0026 & 0.003 & 0.006 & 0.007 \\
\hline
\end{tabular}

Anti-lock braking systems (ABS) are meant to control the wheel slip in order to maintain the friction coefficient close to the optimal value[3][11]. Wheel slip is defined as 
the relative motion between a wheel (tire) and the surface of the road, during vehicle movement. Wheel slip occurs when the angular speed of the wheel (tire) is greater or less compared to it's free-rolling speed[7][8].

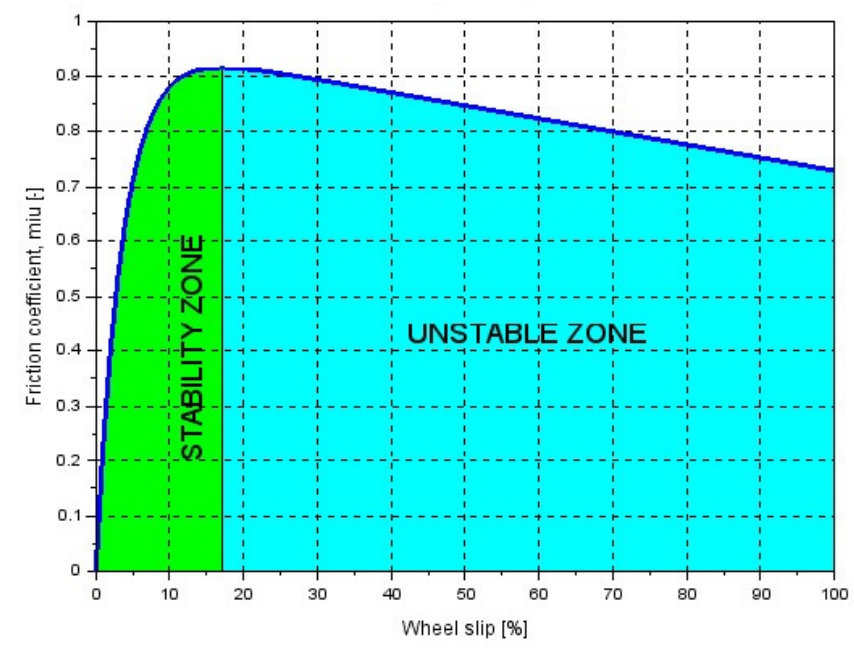

Figure 1. mu vs slip on concrete surface.[8]

During braking, if the wheel slip is $100 \%$ the wheel is locked but the vehicle is still moving. At $0 \%$ slip, the wheel and vehicle have e the same speed.

In Figure 1 the optimum friction coefficient (highest value) is obtained when the wheel slip is around $20 \%$. As you can see, the friction coefficient curve is split into two areas:

- stability zone: where the friction coefficient increases with the increase in wheel slip[8].

- unstable zone: where the friction coefficient decreases with the increase in wheel slip[8].

If the wheel slip enters an unstable area, the friction coefficient will decrease and the wheel will lock causing skid and vehicle instability.

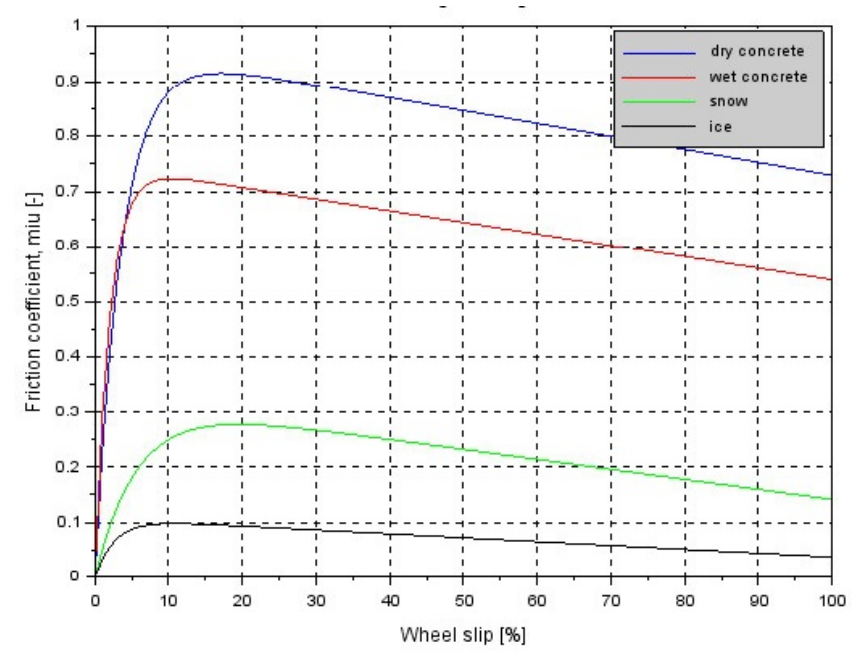

Figure 2. mu vs slip on different surface.[8]
In Figure 2, it can be seen that the maximum value of the friction coefficient decreases sharply for a road covered by snow or ice. Even if the value of the friction coefficient is not significantly lower for $100 \%$ slip, preventing wheel lock improves vehicle maneuverability (steering)[1][3].

\section{Electronic Stability Control}

An electronic control unit of the vehicle is responsible to maintain the stability, traction of vehicle with the road, to prevent roll-over and to reduce side slip. This will be helpful in dangerous scenarios like slippery roads or braking during a steep turn at a very high speed[2].

\subsection{Antilock Braking System}

ABS is the system which assists the driver while applying a brake. For our study to tune the ABS controller for better efficiency, we have worked on three parameters of ABS given by CarSim - Slip ON , Slip OFF and Cut-off speed[7][8][9].

- Slip ON : This is the wheel slip condition at which brakes are turned ON by the ABS controller where ' 0 ' slip represents free rolling and ' 1 ' represents full lockup[7][8][9].

- Slip OFF : This is the wheel slip condition at which brakes are turned OFF by the ABS controller where ' $O$ ' slip represents free rolling and ' 1 ' represents full lockup[7][8][9].

- Cut-OFF speed : It is the minimum wheel speed for the built in ABS controller. When absolute vehicle speed drops below this level the ABS controller is turned off and wheel lockup is allowed. [7][8][9]

\subsection{Analysis and Physics}

$$
w_{v}=\frac{V}{R}
$$

$\mathrm{V}=$ Vehicle linear velocity

$\mathrm{R}=$ Wheel Radius

$w_{v}=$ Vehicle speed divided by wheel radius from (Equation 2)

$w_{w}=$ wheel angular velocity

$$
\text { slip }=1-\frac{w_{w}}{w_{v}}
$$

\section{Test Cases}

In our test we have used the same vehicle as given in section 2 with and without ABS controller for two scenarios. The two situations are as follows:- 


\subsection{Split mu}

In this case there are two different coefficients of friction on the same road. Hence wheels of vehicle can lose traction and make the vehicle unstable which can lead to accidents. These situations can occur if the roads are not constructed properly or there is oil on certain parts of the road, or during rainy days as given in Equation 1, Table 1 [4][5].

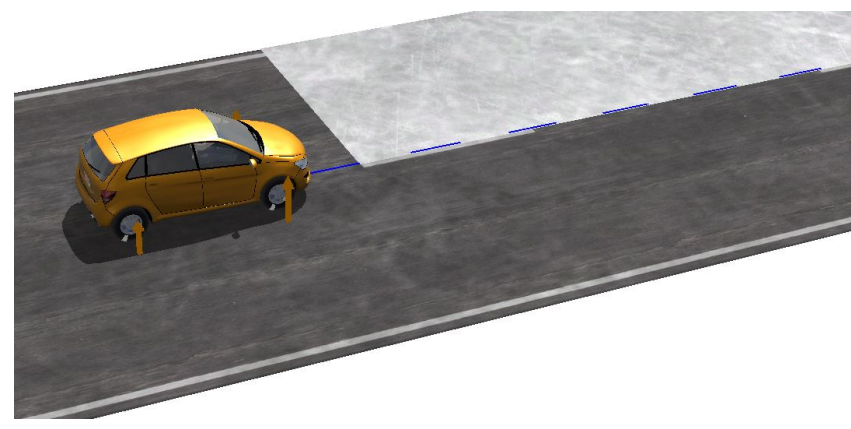

Figure 3. On Road Split-mu

\subsection{Tight turning point of the road}

This is a situation where tight turns are involved and brakes are applied to prevent the vehicle from going off the road[2][5].

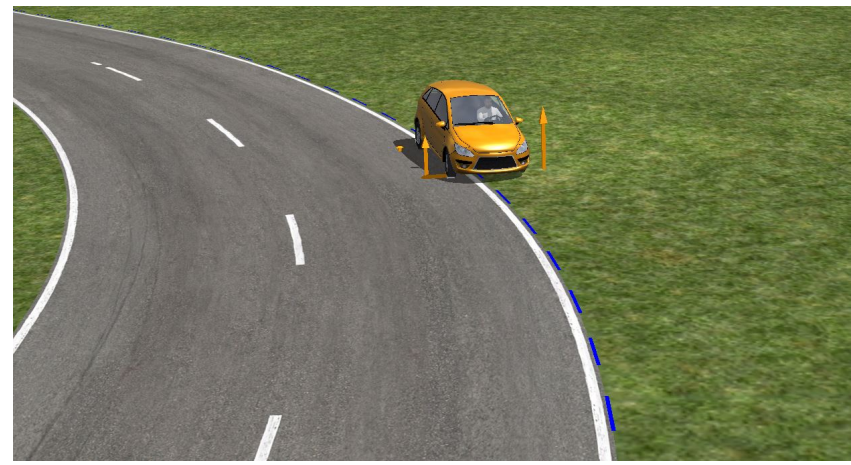

Figure 4. Tight turning point of the road

\section{Results}

We are testing this vehicle for 2 conditions on the road from section 5. First is Split-mu condition from Figure 3. In this condition the coefficient of friction is different for different wheels in the vehicle and the condition can be a case when oil is spilled on the road or road is slippery on some parts so all the wheels do not get proper traction. Hence we have simulated this condition using Carsim and the results are given below with and without Abs system from section $2,4,5$.

\subsection{Split-mu Results}

In the Figure 5 the misbalance of the vehicle can be clearly observed. As ABS is not present in the vehicle in this particular scenario hence as we can observe from graph the movement of center of gravity of the vehicle is not linear and it depicts that the vehicle is not stable and in spinning motion.

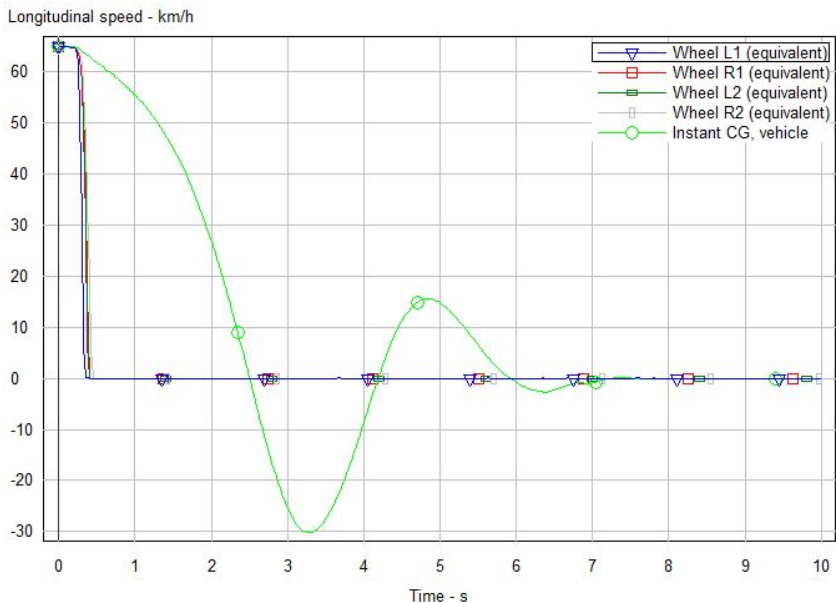

Figure 5. Center of Gravity of the vehicle without ABS.

From Figure 6 when it is compared to previous response of Figure 5 with no ABS it has a better stability and has thus prevented the misbalance of Center of Gravity of the vehicle. This is concluded on the basis of linear motion of Center of Gravity of the vehicle.

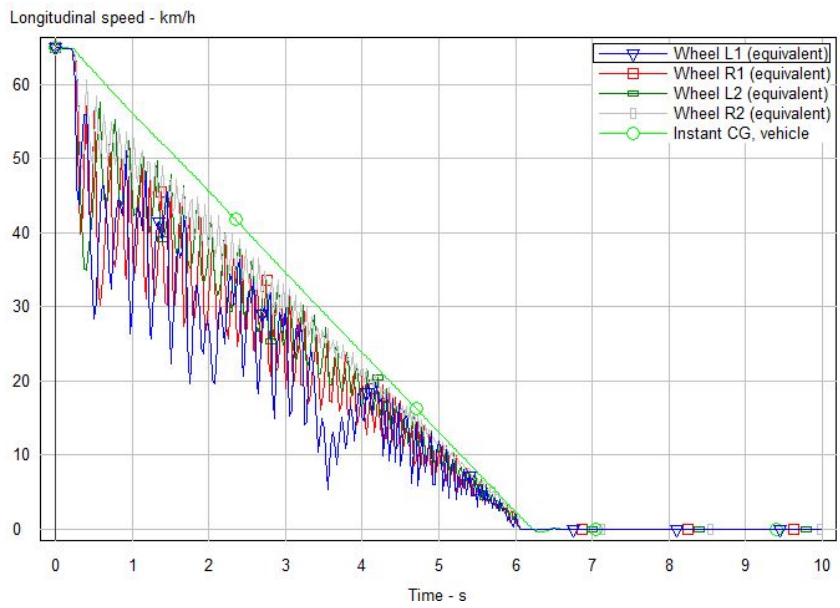

Figure 6. Center of Gravity of the vehicle with Abs.

In the Figure 7 it can be clearly seen that without ABS, brake pressure on wheels becomes constant and wheels gets locked which lead to wheel slip from section 3 this results in the misbalance of the vehicle and it may lead to accidents[3]. 


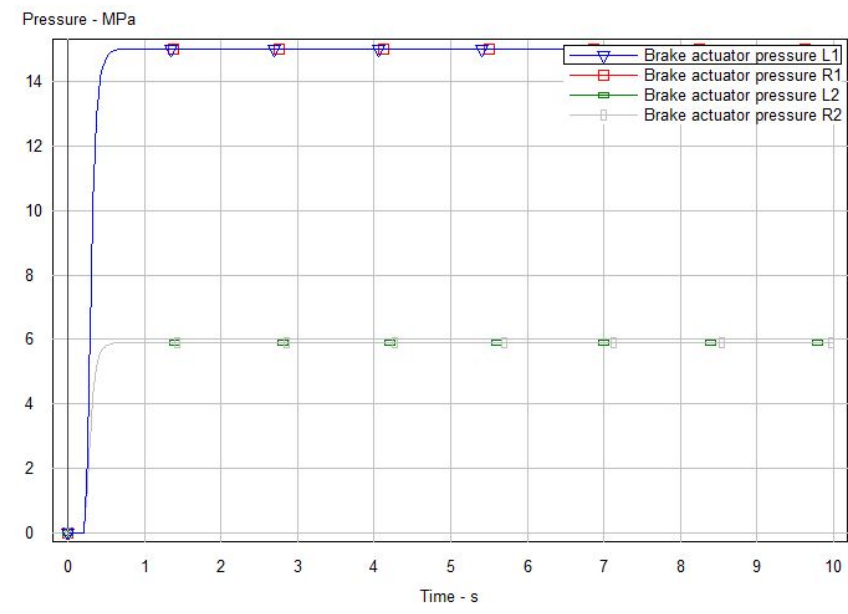

Figure 7. Brake Pressure on the brake cylinders of the wheels without ABS.

In Figure 8 we can observe that vehicle with ABS controller, controls the brake pressure and prevents the wheels from getting locked. From section 3 and from Figure 8 it is concluded that ABS has maintained slip around $20 \%$ and thus it has maintained the stability of the vehicle and the value of maintaining slip at $20 \%$ was refered from Figure 1.

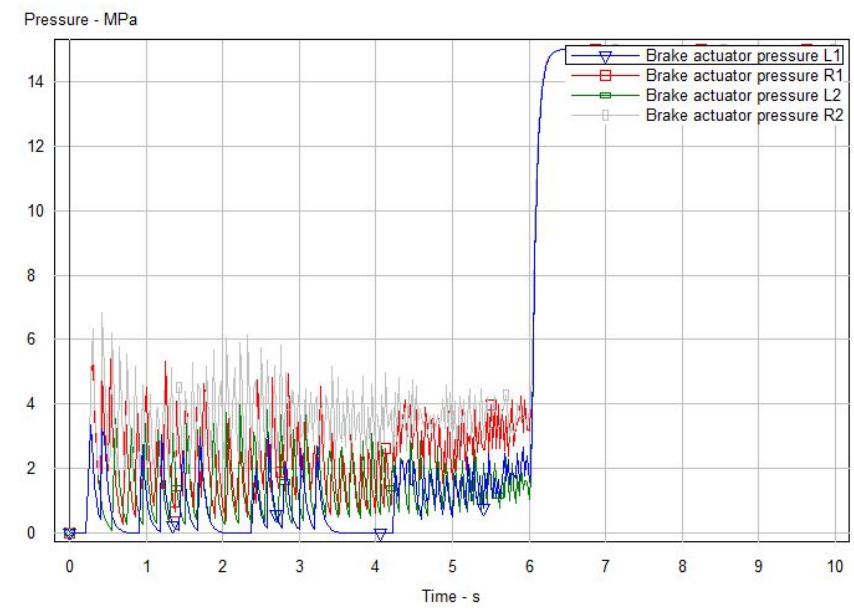

Figure 8. Brake Pressure on the brake cylinders of the wheels with ABS.

From the Figure 9 vehicle without ABS controller, a slip is observed in both the directions as from Figure 5 it gave us the response for the spining motion. As the plot shows slip value to be '1' it indicates the full lock-up of the wheels and negative and positive sign just indicates at the direction of slip, thus it lead to instability and may cause accidents[8].

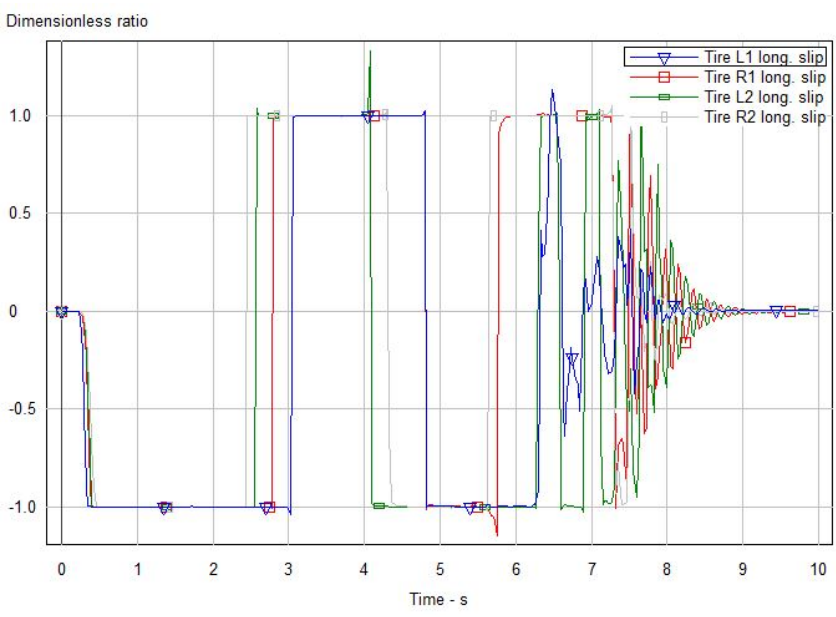

Figure 9. Tire Slip without ABS.

In the Figure 10 , vehicle with a ABS controller, slip is maintained around 0.2 ie $20 \%$ of wheel slip from Figure 1 , until the speed of the vehicle does not come below the threshold value to prevent full lock-up off the wheels so that vehicle stability is maintained[8].

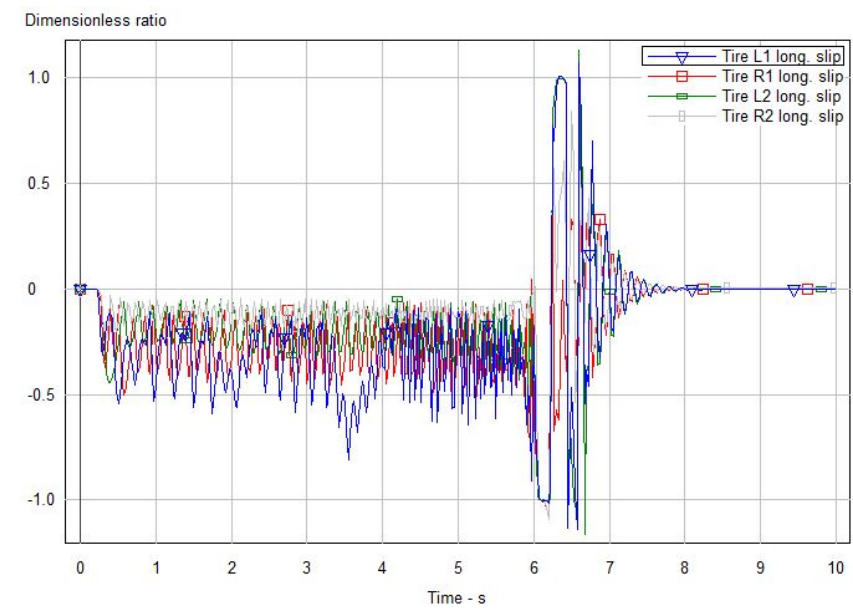

Figure 10. Tire Slip with ABS.

\subsection{Simulink Interface}

The Vehicle model has been implemented on MATLAB simulink interface and the corresponding results can be observed from Figure 12, 14. The model is in Simulink can be interfaced with various external hardwares and it increases the scope of development which make CarSim more powerfull along with Matlab Simulink.[9][10].

In Figure 11 the simulink model is shown where CarSim S-function block is given by Carsim software which has all the parameters of vehicle and as Figure 11 is the model that represent without ABS there is no ABS controller block and at the Brake Actuator Block control mode input is constant 1. This mean that full brake pressure is applied and as we have seen in section 3 this condition leads to wheel lock. 


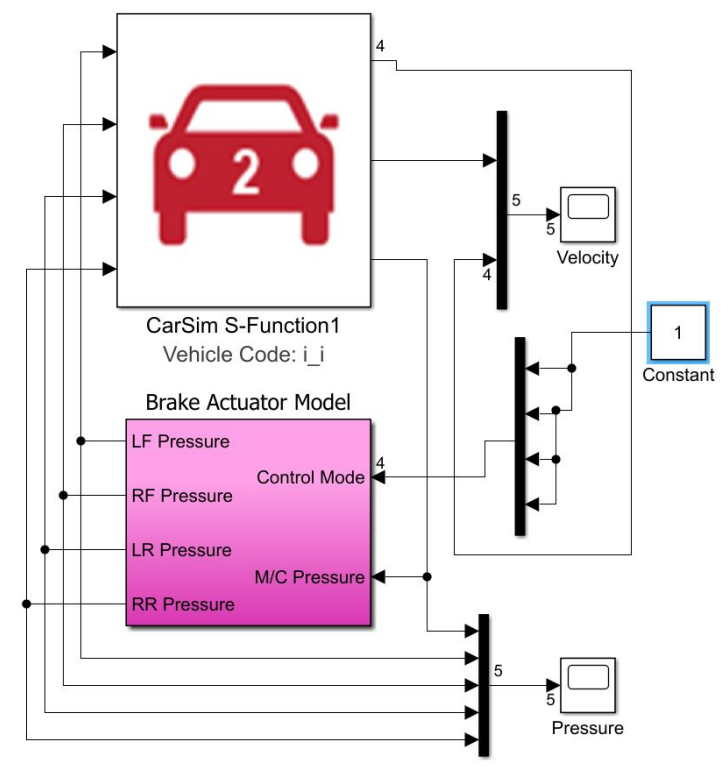

Figure 11. Simulink Model Without ABS Controller.

From Figure 12 the corressponding response of Center of Gravity of the Vehicle is scene which is same as observed in Figure 5 and it has been explained in section 6 both of the results are same because ABS was not present in both condition just the difference is that Figure 5 is from CarSim and Figure 12 is from simulink scope.

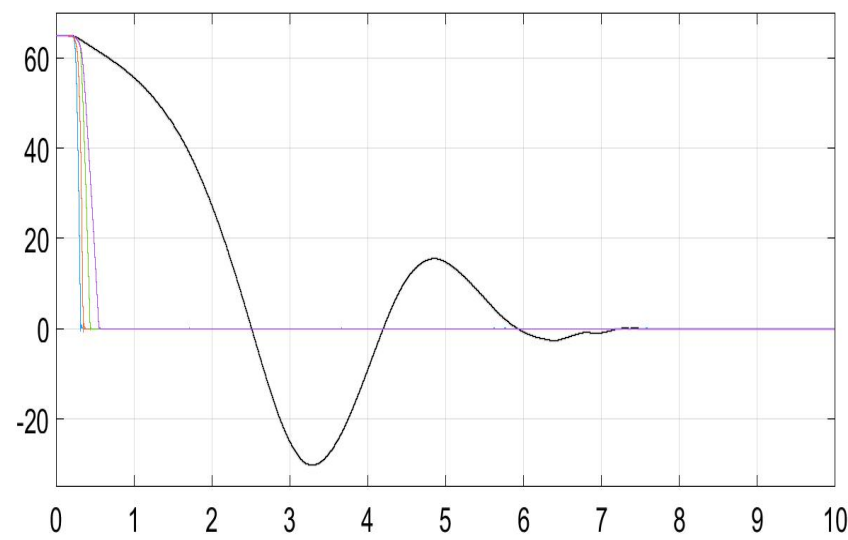

Figure 12. CG of Vehicle Observed in Simulink.

From Figure 13 as you compare it with Figure 11 you can see the presence of ABS controller block which has the data of slip on, off and cut-off as given in section 4. This block work towards maintaining stability as per Figure 2 and controls the slip of the wheels.

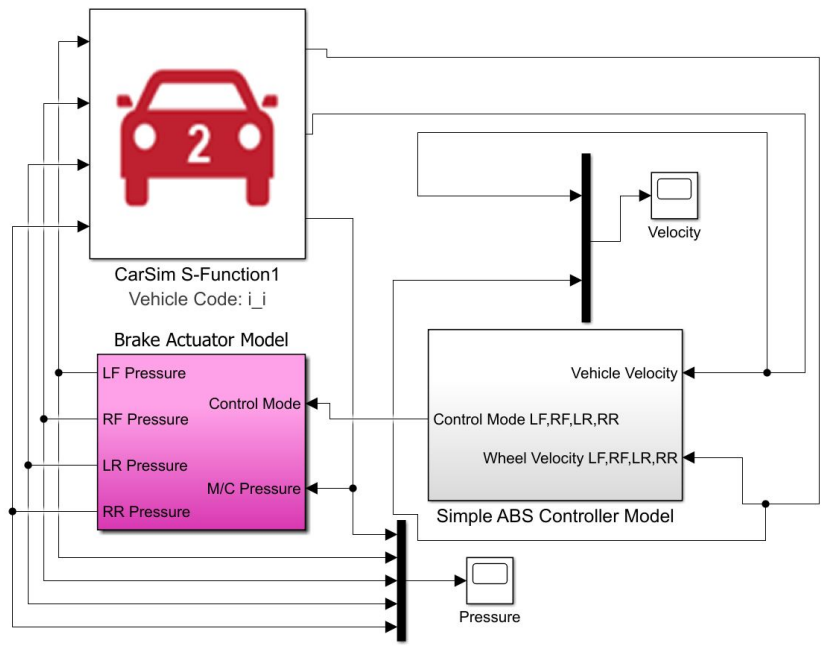

Figure 13. Simulink Model With ABS Controller.

From Figure 14 the corressponding response of Center of Gravity of the Vehicle is scene which is same as observed in Figure 6 and it has been explained in section 6 both of the results are same because ABS is present in both condition just the difference is that Figure 6 is from CarSim and Figure 14 is from simulink scope.

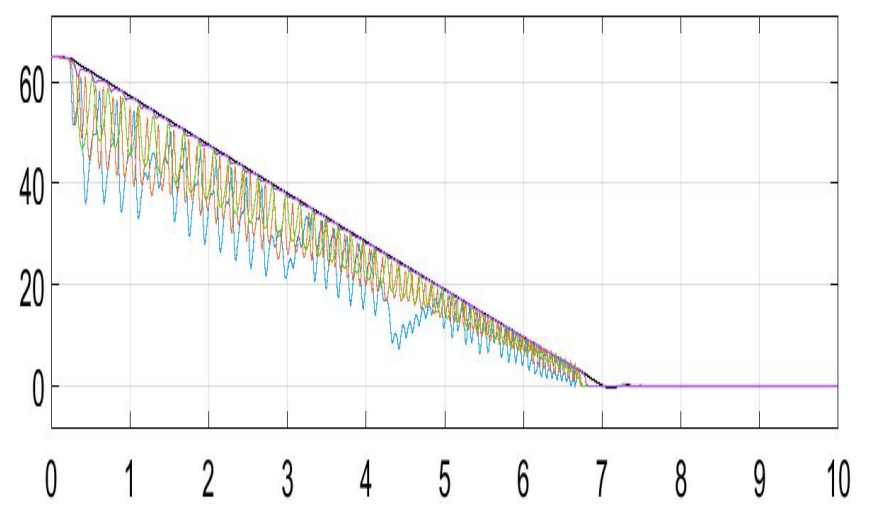

Figure 14. CG of Vehicle Observed in Simulink.

\subsection{Results of tight turning point on the road}

We are testing this vehicle for 2 conditions on the road as given in section 5. Second is Tight turning point of the road condition as shown in Figure 4. In this condition at high speed there are chances of vehicle Rollover[1][6] This can be the condition where driver takes the sudden turn and applies brake to stop the vehicle if it becomes unstable. Hence we have simulated this condition using Carsim and the results are given below with and without Abs system using section 2, 4, 5 .

In Figure 15 the Center of gravity of the vehicle seems to be linear but as we can observed wheel longitudinal speed has suddenly came to 0 which means that the wheels are getting locked and as per section 3 wheels are now slipping and it can take the vehicle off the road 


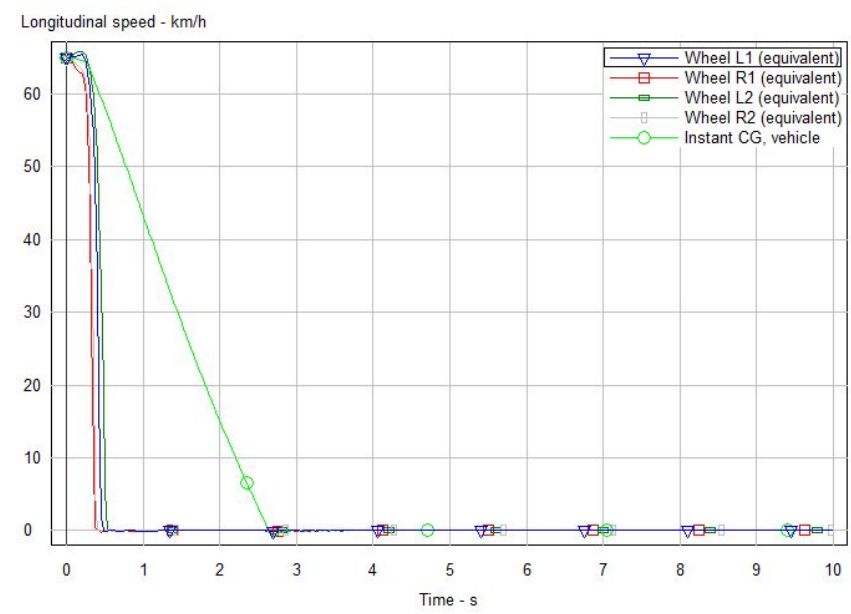

Figure 15. Vehicle Center of Gravity without Abs.

From Figure 16 as we compare it with Figure 15 here the Center of Gravity of the vehicle also seems to be linear but as you can observe the response of the wheels it is not getting locked and as per section 3 vehicle stability is maintained by ABS as it is controlling the slip of the wheels

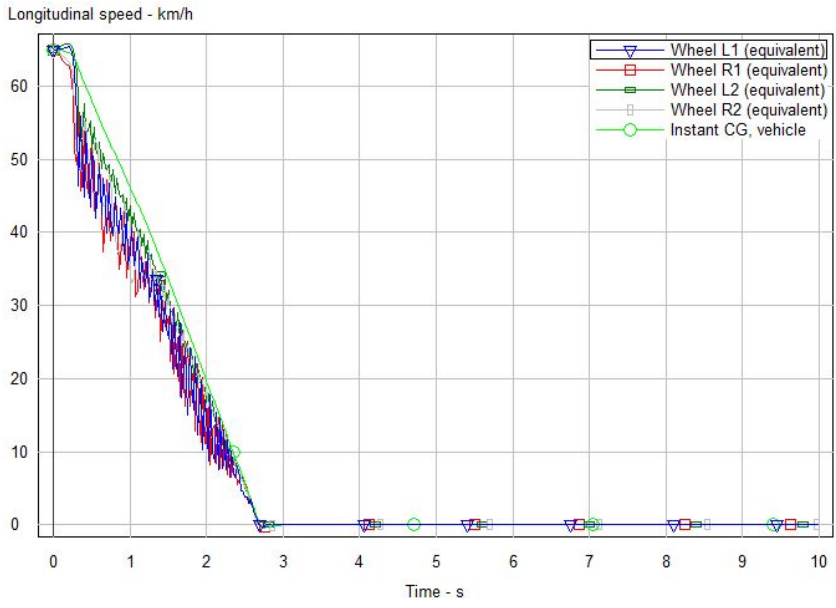

Figure 16. Vehicle Center of Gravity with Abs.

From Figure 15 it was observed that wheels were getting locked which was leading to instability of the vehicle and the reason was that constant brake pressure is applied in the vehicle without ABS which can be observed in Figure 17 and as per section 3 these are the condition for instability

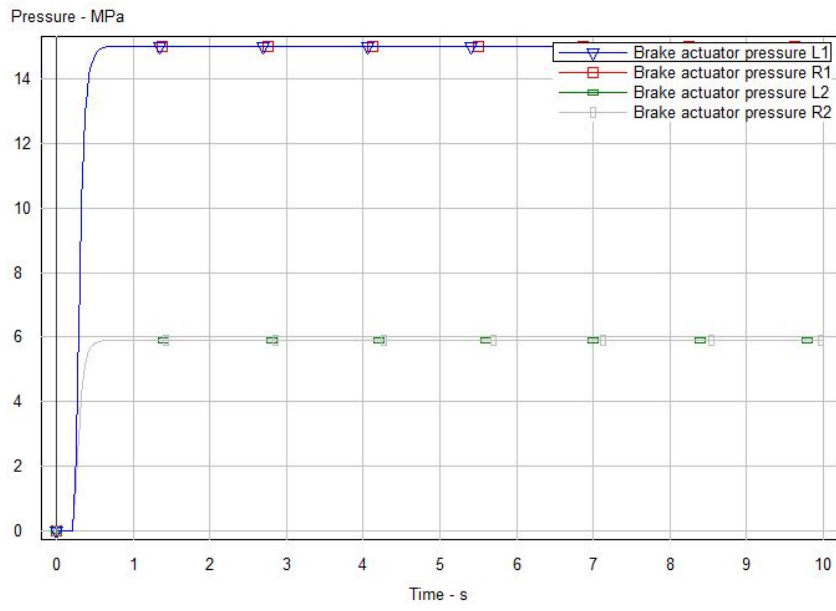

Figure 17. Brake Pressure on the brake cylinders of the wheels without Abs.

From Figure 16 it was observed that wheels were prevented from getting locked which has lead to the stability of the vehicle and the reason was that the brake pressure is controled by ABS according to section 4. The vehicle brakes with ABS response can be observed in Figure 18 this response is trying to maintain wheel slip around $20 \%$ and as per section 3 these are the condition for stability[4]

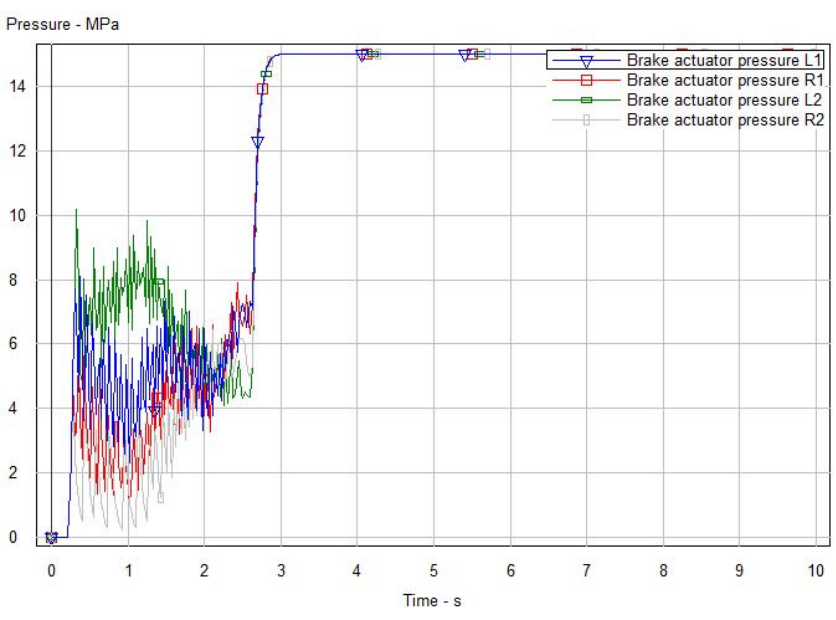

Figure 18. Brake Pressure on the brake cylinders of the wheels With Abs.

In the Figure 19 the complete slip of the tire is observed as the value reaches ' 1 ' when the brakes are applied. The negative and positive sign indicates the direction and as slip is reaching 1 it means wheels are getting locked and this is leading to the instability of vehicle as per section 3[4]. 


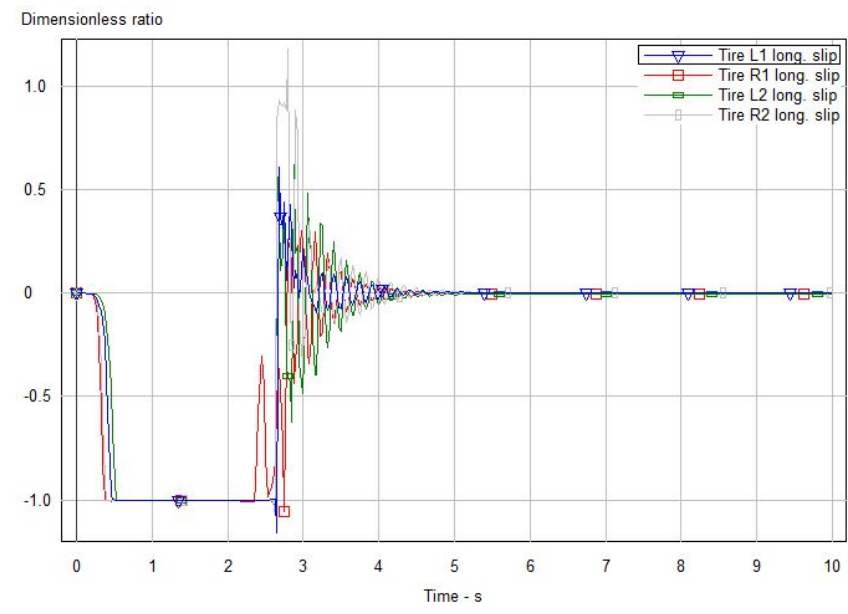

Figure 19. Tire Slip without ABS.

In the Figure 20 the slip of the tire is observed and the value is maintained around 0.2 ie $20 \%$ wheel slip. The negative and positive sign indicates the direction and as vehicle speed decreases considerably and it goes below threshold speed at which wheel lock is safe ie from section 4.1 the Cut-Off speed is that threshold parameter and as wheel lock is allowed it makes the vehicle stationary and keeps the vehicle stable 3[4].

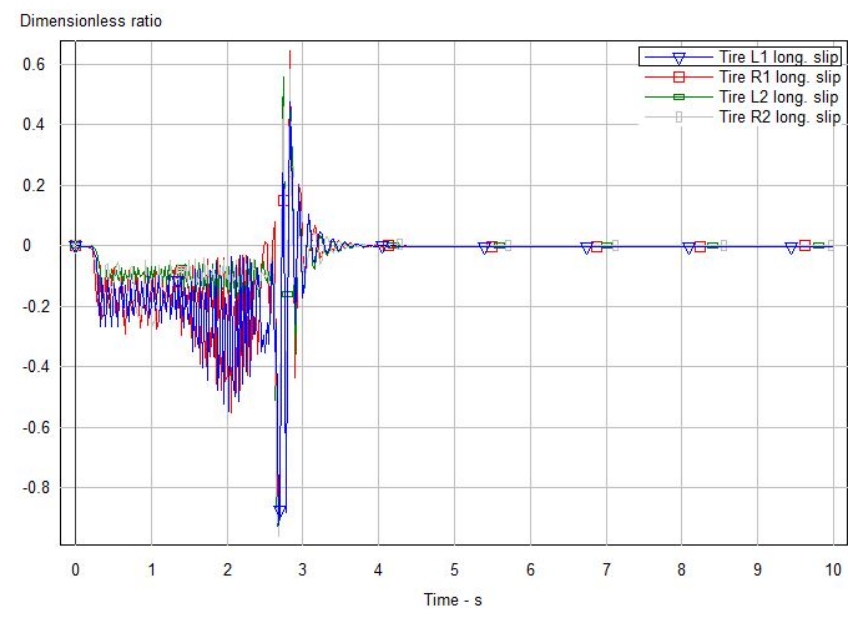

Figure 20. Tire Slip with ABS.

\section{Conclusion}

Carsim can be used for wide variety of simulation and realistic response can be observed using it. The analysis of Electronic Control Unit has been done using Carsim and Simulink. Hence using the tools provided by CarSim all parameters of the vehicle can be studied and system with best effeciency can be developed and this makes it easier to understand dynamics of the vehicle without having a deep mathematical knowledge of it.

\section{Future Scope}

The Hardware-in-the-Loop (HIL) simulation of corresponding vehicle model can be implemented using dSPACE and DSP processor.The vehicle model can be implemented on dSPACE and implementation of controller on DSP processor.

\section{References}

[1] Model-Based Design of a SUV AntiRollover Control System https://www. mathworks.com/matlabcentral/fileexchange/ 23622-model-based-design-of-a-suv-anti-rollover-control-system, MATLAB Central File Exchange.

[2] Observer-Based Robust Control of Vehicle Dynamics for Rollover Mitigation in Critical Situations Hamid Dahmani, Olivier Pagès, Ahmed El Hajjaji, and Nawal Daraoui

[3] New Paradigms for the Integration of Yaw Stability and Rollover Prevention Functions in Vehicle Stability Control Rajesh Rajamani, Senior Member, IEEE, and Damrongrit (Neng) Piyabongkarn, Member, IEEE

[4] Switched stable control design methodology applied to vehicle rollover prevention based on switched suspension settings

[5] Driving Control Algorithm for Maneuverability, Lateral Stability, and Rollover Prevention of 4WD Electric Vehicles With Independently Driven Front and Rear Wheels Juyong Kang, Jinho Yoo, and Kyongsu Yi

[6] A Three-Dimensional Dynamics Control Framework of Vehicle Lateral Stability and Rollover Prevention via Active Braking With MPC Liang Li, Member, IEEE, Yishi Lu, Rongrong Wang, and Jie Chen

[7] Modeling an Anti-Lock Braking System https://www.mathworks.com/help/simulink/slref/ modeling-an-anti-lock-braking-system.html

[8] https://x-engineer.org/projects/ anti-lock-braking-system-abs-modeling-simulation-xcos

[9] https://www.carsim.com/downloads/pdf/CarSim Math_Models.pdf

[10] https://www.carsim.com/products/supporting/ simulink/index.php

[11] An empirical study of the effectiveness of electronic stability control system in reducing loss of vehicle control Yiannis E. Papelis ,1, Ginger S.Watson2, Timothy L. Brown The University of Iowa, Iowa City, IA 52242, United States 